\title{
Clinical outcomes and prognostic factors in patients with mycosis fungoides who underwent radiation therapy in a single institution
}

\author{
Bum-Sup Jang, MD¹, Eunji Kim, MD¹, II Han Kim, MD, PhD ${ }^{1,2}$, Hyun-Cheol Kang, MD, PhD, \\ Sung-Joon Ye, MD, PhD \\ 'Department of Radiation Oncology, Seoul National University Hospital, Seoul; \\ ${ }^{2}$ Institute of Radiation Medicine, Cancer Research Institute, Seoul National University College of Medicine, Seoul; \\ ${ }^{3}$ Program in Biomedical Radiation Sciences, Department of Transdisciplinary Studies, Graduate School of Convergence Science \\ and Technology, Seoul National University, Seoul, Korea
}

Purpose: We aimed to evaluate clinical outcomes including progression-free survival (PFS), overall survival (OS), partial response, and complete response in patients who underwent radiation therapy (RT) for mycosis fungoides (MF). Also, we sought to find prognostic factors for clinical outcomes.

Materials and Methods: Total 19 patients confirmed with MF between 1999-2015 were retrospectively reviewed. Clinical and treatment characteristics, clinical outcomes, and and toxicities were analyzed.

Results: Eleven patients were treated with total skin electron beam radiotherapy (TSEBT) and 8 patients with involved field radiation therapy (IFRT) with median dose of $30 \mathrm{~Gy}$, respectively. The median time interval from diagnosis to RT was 2.6 months (range, 0.4 to 87.3 months). The overall response rate was 100\%; 11 patients (57.9\%) had a complete response and 8 patients (42.1\%) a partial response. The presence of positive lymph node at the time of consultation of RT was associated with lower OS ( $p=0.043)$. In multivariate analysis, PFS was significantly lower for patients with increased previous therapies experienced following RT ( $p=$ $0.019)$ and for patients showing PR during RT ( $p=0.044)$. There were no reported grade 3 or more skin toxicities related with RT.

Conclusion: Both IFRT and TSEBT are effective treatment for MF patients. Patients with short disease course before RT or complete response during RT are expected to have longer PFS. Positive lymph node status at the initiation of RT was associated woth poor OS, suggesting other treatment modalities such as low-dose RT for patients with low life-expectancy.

Keywords: Mycosis fungoides, Radiotherapy, Total skin electron beam radiotherapy, Cutaneous T-cell lymphoma, Total skin electron irradiation

\section{Introduction}

Mycosis fungoides (MF) is the most common type of non-
Hodgkin T-cell lymphoma with major presentation in skin. It accounts for only about $4 \%$ of all cases of non-Hodgkin lymphoma. Median survival ranges from less than 11 months

Received 28 November 2017, Revised 30 December 2017, Accepted 18 January 2018.

Correspondence: II Han Kim, MD, PhD, Department of Radiation Oncology, Seoul National University College of Medicine, 103 Daehak-ro, Jongno-gu, Seoul 03080, Korea. Tel: +82-2-2072-2528, Fax: +82-2-765-3317, E-mail: ihkim@snu.ac.kr

(c) This is an Open Access article distributed under the terms of the Creative Commons Attribution Non-Commercial License (http://creativecommons.org/ licenses/by-nc/4.0/) which permits unrestricted non-commercial use, distribution, and reproduction in any medium, provided the original work is properly cited.

www.e-roj.org 
to greater than 24 years according to clinical stage $[1,2]$. Clinical stage is based on the TNMB classification indicating the involvement of skin (T), lymph nodes $(\mathrm{N})$, viscera $(\mathrm{M})$, and blood (B) stage [3], respectively. This staging system is based on the International Society for Cutaneous Lymphomas (ISCL)/ European Organization for Research and Treatment of Cancer (EORTC) revised staging system.

For most MF patients involving the limited percent body surface area, involved field radiation therapy (IFRT) is suggested. Since MF is sensitive to radiation therapy (RT), ionizing radiation is effective treatment modality for localized and early stage MF $[4,5]$. In case of unilesional MF tumors, IFRT with the doses as low as 8 Gy can be delivered, achieving long-term local control [6]. Localized RT have shown overall response (OR) rate of 100\% and complete response (CR) rate of more than 90\% [7]. On the other hand, when lesions are widespread in whole body or resistant to previous therapies, total skin electron beam radiotherapy (TSEBT) can be considered. Conventional doses of TSEBT are ranges from 30 to $36 \mathrm{~Gy}$, showing good CR rate (95\%-100\%) [8,9]. Also, TSEBT can provide palliative effect to patients with advanced MF [10].

Most MF studies have been performed mainly in Western countries. In contrast, there are few studies reporting clinical pattern and outcomes of MF in Asia. This is probably due to the high incidence of cutaneous natural killer/T-cell lymphomas rather than MF. Thus, in this retrospective study, we aimed to analyze clinical responses, outcomes and toxicities of TSEBT or IFRT in patients with advanced or refractory disease of MF. Additionally, progression-free survival (PFS) and overall survival (OS) rates were estimated.

\section{Materials and Methods}

\section{Patients}

A total 19 patients, who were pathologically confirmed with MF and received RT in Seoul National University Hospital from July 1999 to December 2015, were collected and reviewed. Baseline patient, tumor characteristics, adverse effects, responses during treatment, and treatment outcomes including PFS and OS were reviewed. Since patients who had visceral organ involvement or had significant blood involvement were excluded in the present study, we could only identify $T$ and $\mathrm{N}$ stage at the time of consultation for RT among the TNMB staging components. This study is approved by Institutional Review Board of Seoul National University Hospital (No. H-1708-063-877).

\section{Radiation therapy technique}

For TSEBT, a modified Stanford technique was used to deliver TSEBT with doses ranged from 20 to $36 \mathrm{~Gy}$ (median, $30 \mathrm{~Gy}$ ) in median 20 fractions (range, 10 to 36 fractions). Fraction size was ranged from 1.0 to $2.0 \mathrm{~Gy}$, and one fraction consisting of all 6 positions and 12 fields were treated over 2 days. Thus, in each RT session, radiation was delivered to 6 positions and 3 fields. After 2 consecutive days of RT session, 1 day was rested resulting in four $\mathrm{RT}$ sessions in a week with resting on Wednesday. Six MeV or $9 \mathrm{MeV}$ electron beam which field size was $34 \mathrm{~cm} \times 34 \mathrm{~cm}$ was used to treat three anterior (anteriorposterior $[\mathrm{AP}]$, left anterior oblique [LAO], and right anterior oblique [RAO]) and three posterior (posterior-anterior [PA] left posterior oblique [LPO], and right posterior oblique [RPO]) treatment fields. The spoiler, a home-made acrylic plate of $106 \mathrm{~cm} \times 205 \mathrm{~cm} \times 1 \mathrm{~cm}$, was placed $350 \mathrm{~cm}$ distant from the isocenter and positioned $30 \mathrm{~cm}$ from the skin. Gantry was adjusted with angle of $90^{\circ} \pm 18^{\circ}$ in order to obtain the dual field that is perpendicular to the treatment plane. During RT, patients should be stand in an upright position without movement, and they are placed on the wooden platform of $20 \mathrm{~cm}$ height to reduce scatter from the floor. Based on in vivo dosimetry measurement, all patients who underwent TSEBT $(n=11)$ should have been undertaken additional boost $\mathrm{RT}$ in order to compensate shadowed body region including scalp, palm, sole, axillary or to control a large lesion which were not dramatically responding during planned treatment session. Boost doses were ranged from 5 to 9 Gy depending on radiation oncologist's discretion. Typically, $6 \mathrm{MeV}$ electron beam was used to compensate those shadowed areas with 1 $\mathrm{cm}$ of bolus to deliver $90 \%$ of the prescribed dose in openfield.

For IFRT, either one of electron beam ( $6 \mathrm{MeV}$ or $9 \mathrm{MeV}$ ) or photon beam ( $6 \mathrm{MV}$ or $10 \mathrm{MV}$ ) were used to deliver doses ranged from 18 to $45 \mathrm{~Gy}$ (median, $36 \mathrm{~Gy}$ ) with 6-25 fractions. IFRT was delivered to skin lesions with a margin of at least 1.5 $\mathrm{cm}$ of normal skin. In IFRT group, one patient was treated with combination with both electron and photon beam.

\section{Evaluation of response and toxicities}

We reviewed and evaluated the response using Physician's Global Assessment (PGA) method which used in the T-cell lymphoma study [11]. During TSEBT or IFRT, the response was evaluated weekly. CR was defined as complete regression of visible skin lesions, and partial response (PR) as 50\%-99\% clearance of skin disease from baseline without new tumor. Progression was defined as disease recurrence within radiation 
field, in regional lymph nodes, or distant metastasis. Acute toxicities such as skin reaction, edema, or blisters during RT sessions were reviewed according to Common Terminology Criteria for Adverse Events version 4.0.

\section{Statistics}

Patient and tumor characteristics according to RT responses were compared using chi-square test. PFS was calculated from the initiation of RT to disease progression including locoregional recurrence, distant metastasis or death. OS was defined as time interval from diagnosis to last follow-up or death. Survival curves for PFS and OS were depicted using Kaplan-Meier methods, and log-rank tests were performed to compare PFS and OS rates between groups. Prognostic

Table 1. Patient and treatment characteristics stratified by RT responses

\begin{tabular}{|c|c|c|c|c|}
\hline Characteristic & $P R(n=8)$ & $C R(n=11)$ & Total $(n=19)$ & $p$-value $e^{a)}$ \\
\hline Age (yr) & & & & 0.096 \\
\hline$\leq 60$ & $6(75.0)$ & $4(36.4)$ & $10(52.6)$ & \\
\hline$>60$ & $2(25.0)$ & $7(63.6)$ & $9(47.4)$ & \\
\hline Gender & & & & 0.260 \\
\hline Female & $5(62.5)$ & $4(36.4)$ & $8(42.1)$ & \\
\hline Male & $3(37.5)$ & $7(63.6)$ & $11(57.9)$ & \\
\hline Stage at diagnosis & & & & 0.071 \\
\hline $\mathrm{IB}$ & $2(25.0)$ & $1(9.1)$ & $3(15.8)$ & \\
\hline$\| A$ & $0(0)$ & $1(9.1)$ & $1(5.3)$ & \\
\hline$\| B$ & $3(37.5)$ & $9(81.8)$ & $12(63.2)$ & \\
\hline IIIA & $3(37.5)$ & $0(0)$ & $3(15.8)$ & \\
\hline Lymph node status & & & & 0.636 \\
\hline Clinically not detected & $5(62.5)$ & $8(72.7)$ & $13(68.4)$ & \\
\hline Clinically detected & $3(37.5)$ & $3(27.3)$ & $6(31.6)$ & \\
\hline Previous treatment & & & & 0.361 \\
\hline No & $2(25.0)$ & $5(45.5)$ & $7(36.8)$ & \\
\hline Yes & $6(75.0)$ & $6(54.5)$ & $12(63.2)$ & \\
\hline Number of previous therapies & & & & 0.599 \\
\hline $0-1$ & $6(75.0)$ & 7 (63.6) & $13(68.4)$ & \\
\hline $2-3$ & $2(25.0)$ & $4(36.4)$ & $6(31.6)$ & \\
\hline Time interval between diagnosis and RT (mo) & & & & 0.061 \\
\hline$\leq 2$ & $1(12.5)$ & $6(54.5)$ & $7(36.8)$ & \\
\hline$>2$ & $7(87.5)$ & $5(45.5)$ & $12(63.2)$ & \\
\hline Type of RT & & & & 0.198 \\
\hline IFRT & $2(25.0)$ & $6(54.5)$ & $8(42.1)$ & \\
\hline TSEBT & $6(75.0)$ & $5(45.5)$ & $11(57.9)$ & \\
\hline RT dose (Gy) & & & & 0.845 \\
\hline$\leq 30$ & $4(50.0)$ & $6(54.5)$ & $10(52.6)$ & \\
\hline$>30$ & $4(50.0)$ & $5(45.5)$ & $9(47.4)$ & \\
\hline Adjuvant treatment & & & & 0.912 \\
\hline Administered & $6(75.0)$ & $8(72.7)$ & $14(73.7)$ & \\
\hline Not administered & $2(25.0)$ & $3(27.3)$ & $5(26.3)$ & \\
\hline Retreatment following RT & & & & 0.061 \\
\hline No & $1(12.5)$ & $6(54.5)$ & $7(36.8)$ & \\
\hline Yes & 7 (87.5) & $5(45.5)$ & 12 (63.2) & \\
\hline Clinical T stage & & & & 0.071 \\
\hline $\mathrm{T} 1$ & $0(0)$ & $1(9.1)$ & $1(5.2)$ & \\
\hline T2 & $2(25.0)$ & $1(9.1)$ & $3(15.8)$ & \\
\hline T3 & $3(37.5)$ & $9(81.8)$ & $12(63.2)$ & \\
\hline T4 & $3(37.5)$ & $0(0)$ & $3(15.8)$ & \\
\hline
\end{tabular}

Values are presented as number (\%).

$\mathrm{PR}$, partial response; $\mathrm{CR}$, complete response; RT, radiation therapy; IFRT, involve field radiation therapy; TSEBT, total skin electron beam radiotherapy.

${ }^{a} p$-value was estimated using chi-square test. 
factors were explored using univariate and multivariate Cox proportional-hazards regression. Spearman rank correlation coefficient (Spearman rho) was estimated to investigate the relationship between prescription doses and the treatment year. Logistic regression analysis was performed to examine the association between RT doses and responses. All statistical analyses were performed with STATA software version 14 (StataCorp, College Station, TX, USA).

\section{Results}

\section{Patient and treatment characteristics}

Eleven males and eight females were treated with TSEBT or IFRT. Of 19 patients, 11 patients underwent TSEBT and 8 patients did IFRT. The median follow-up was 50.1 months (range, 8.7 to 179.8 months), and median age was 59 years (range, 35 to 75 years). The median time interval between diagnosis to RT was 2.6 months (range, 0.4 to 87.3 months). At the time of consultation for RT, 3 patients had stage IB, $1 \mathrm{IIA}, 12 \mathrm{II} \mathrm{B}$, and $3 \mathrm{III}$ of disease, and 6 patients (31.6\%) had clinically abnormal palpable or detected by computed tomography nodes (Table 1). Among patients with IFRT, two patients showed lymph node positive. When one patient was planning to undergo IFRT to right eyelid lesion, he incidentally found a palpable lymph node involved with MF in right neck level II. Another patient had abdomen wall mass with right inguinal palpable lymphadenopathy. Of 19 patients, 12 patients (63\%) had undergone previous therapies with a median 1 therapy (range, 0 to 4 therapies) including systemic chemotherapy, oral retinoid, or UV-B treatment following RT. Only one patient had terminated TSEBT treatment before boost RT due to interim progression to visceral organ. Followed by completion of RT, 5 patients received adjuvant therapy including systemic chemotherapy $(n=1)$, photodynamic therapy $(n=1), U V-B(n=2)$, and methotrexate $(n=1)$. Twelve patients received salvage treatment due to disease progression, and 3 of them underwent re-RT (Table 2).

\section{Response and clinical outcome}

With the median time to evaluation of 72 days (range, 15 to 134 days), all patients showed OR followed by RT; 11 patients (57.9\%) showed CR and 8 patients (42.1\%) PR. Patient and tumor characteristics stratified by RT responses are shown in Table 1. Patients with $\leq 2$ months of median time to RT had a tendency to achieve a CR rather than PR (54.5\% vs. $12.5 \%$; $p$ $=0.061)$. There was a trend for more retreatment after RT with patients showing PR ( $87.5 \%$ vs. $45.4 \% ; p=0.061)$. There were
Table 2. Previous, adjuvant, and salvage therapies of patients treated with RT

\begin{tabular}{lc}
\hline & No. of cases \\
\hline Previous therapies & \\
Systemic chemotherapy & 5 \\
Oral retinoid & 4 \\
UV-B & 5 \\
MTX & 3 \\
Topical carmustine & 2 \\
Photodynamic therapy & 2 \\
PUVA & 1 \\
Interferon & 1 \\
Topical steroid & 1 \\
Adjuvant therapies & \\
Systemic chemotherapy & 1 \\
Photodynamic therapy & 1 \\
UV-B & 2 \\
MTX & 1 \\
Salvage therapies & \\
Systemic chemotherapy & 5 \\
Re-RT & 3 \\
MTX & 2 \\
Photodynamic therapy & 2 \\
UV-B & 3 \\
\hline RT
\end{tabular}

$R T$, radiation therapy; UV-B, ultraviolet-B; PUVA, psoralen plus ultraviolet $A ;$ MTX, methotrexate.

no significant differences in $\mathrm{RT}$ responses between age groups $(p=0.096)$, gender $(p=0.260)$, initial stages $(p=0.071)$, lymph node status $(p=0.636)$, the receipt of previous therapies $(p=$ $0.361)$, the number of previous therapies $(p=0.599)$, types of RT $(p=0.198)$, radiation doses $(p=0.845)$, and the receipt of adjuvant treatment $(p=0.912)$.

In case of TSEBT, we defined progression as recurrence within the radiation field or distant metastasis. Of 10 patients who received TSEBT, 7 patients experienced recurrence of skin lesion in body, and 3 patients did distant metastasis such as sternum or diffuse lymph node involvement. On the other hand, 2 patients who had received IFRT reported distant metastasis as a disease progression. They all experienced recurrence of another skin lesion except where IFRT was performed. There were no cases of recurrence in regional node.

The overall median PFS was 13.6 months, and the median PFS rate was $46 \%$. PFS rate were not significantly different when patients had been treated with IFRT compared with TSEBT ( $p=0.085)$ (Fig. 1A). In univariate analysis, the increasing number of previous therapy (hazard ratio $[H R]=$ 2.30; 95\% confidence interval $[\mathrm{Cl}], 1.17-4.50 ; p=0.015)$ and $P R(H R=3.63 ; 95 \% C l, 1.11-11.82 ; p=0.032)$ were associated 
Table 3. Univariate analysis for progression-free survival and overall survival

\begin{tabular}{|c|c|c|c|c|c|c|}
\hline & \multicolumn{3}{|c|}{ Progression-free survival } & \multicolumn{3}{|c|}{ Overall survival } \\
\hline & $\mathrm{HR}$ & $95 \% \mathrm{Cl}$ & $p$-value $e^{a)}$ & $\mathrm{HR}$ & $95 \% \mathrm{Cl}$ & $p$-value \\
\hline \multicolumn{7}{|l|}{ Age (yr) } \\
\hline$\leq 60$ & 1.00 & & & 1.00 & & \\
\hline$>60$ & 1.01 & $0.32-3.20$ & 0.981 & 0.70 & $0.17-2.81$ & 0.610 \\
\hline \multicolumn{7}{|l|}{ Gender } \\
\hline Female & 1.00 & & & 1.00 & & \\
\hline Male & 1.01 & $0.32-3.21$ & 0.981 & 0.70 & $0.17-2.81$ & 0.610 \\
\hline \multicolumn{7}{|l|}{ Previous treatment } \\
\hline No & 1.00 & & & 1.00 & & \\
\hline Yes & 2.53 & $0.68-9.50$ & 0.168 & 0.91 & $0.21-3.85$ & 0.894 \\
\hline \multicolumn{7}{|c|}{ Number of previous therapies } \\
\hline $0-1$ & 1.00 & & & 1.00 & & \\
\hline $2-3$ & 3.65 & $1.13-11.75$ & 0.030 & 1.29 & $0.28-5.90$ & 0.744 \\
\hline $0-3$, incremental & 2.30 & $1.17-4.50$ & 0.015 & 1.20 & $0.60-2.41$ & 0.613 \\
\hline \multicolumn{7}{|l|}{ Median time to RT (mo) } \\
\hline$\leq 2$ & 1.00 & & & 1.00 & & \\
\hline$>2$ & 1.72 & $0.46-6.39$ & 0.420 & 1.28 & $0.23-7.06$ & 0.781 \\
\hline Incremental & 1.01 & $0.99-1.03$ & 0.479 & 1.01 & $0.99-1.03$ & 0.479 \\
\hline \multicolumn{7}{|l|}{ Stage at diagnosis } \\
\hline |B-I||A, incremental & 1.01 & $0.47-2.14$ & 0.986 & 1.55 & $0.67-3.58$ & 0.309 \\
\hline \multicolumn{7}{|l|}{ T stage } \\
\hline T1-T4, incremental & 1.79 & $0.62-5.14$ & 0.280 & 2.42 & $0.72-8.12$ & 0.154 \\
\hline \multicolumn{7}{|l|}{ Lymph node status } \\
\hline Clinically negative & 1.00 & & & 1.00 & & \\
\hline Clinically positive & 2.47 & $0.77-7.93$ & 0.129 & 4.88 & $0.89-26.74$ & 0.068 \\
\hline \multicolumn{7}{|l|}{ Type of RT } \\
\hline IFRT & 1.00 & & & 1.00 & & \\
\hline TSEBT & 3.09 & $0.81-11.85$ & 0.100 & 1.35 & $0.32-5.73$ & 0.682 \\
\hline \multicolumn{7}{|l|}{ RT dose (Gy) } \\
\hline$\leq 30$ & 1.00 & & & 1.00 & & \\
\hline$>30$ & 0.69 & $0.22-2.22$ & 0.540 & 1.17 & $0.27-5.04$ & 0.830 \\
\hline \multicolumn{7}{|l|}{ Response } \\
\hline $\mathrm{CR}$ & 1.00 & & & 1.00 & & \\
\hline PR & 3.63 & $1.11-11.82$ & 0.032 & 2.78 & $0.64-12.16$ & 0.174 \\
\hline \multicolumn{7}{|l|}{ Adjuvant treatment } \\
\hline Not administered & 1.00 & & & 1.00 & & \\
\hline Administered & 0.95 & $0.26-3.50$ & 0.934 & 2.87 & $0.46-17.82$ & 0.259 \\
\hline
\end{tabular}

$\mathrm{HR}$, hazard ratio; $\mathrm{Cl}$, confidence interval; $\mathrm{RT}$, radiation therapy; IFRT, involved field radiation therapy; TSEBT, total skin electron beam radiotherapy; $C R$, complete response; $P R$, partial response.

${ }^{a)} p$-value was estimated using Cox hazard-proportional regression.

with the increased risk of disease progression (Table 3). A waterfall plot in Fig. 1B demonstrates PFS for all patients according to RT responses. The majority of patients with $\mathrm{CR}$ had PFS more than 13.6 months that is the median PFS. Fig. 1C and $1 \mathrm{D}$ shows the Kaplan-Meier curves for PFS according to RT responses and the number of previous therapies, respectively. Multivariate analysis stratified by RT modalities (TSEBT vs. IFRT) demonstrated that increasing number of previous therapies ( $p$ $=0.019)$ and partial response $(p=0.044)$ were remained as an https://doi.org/10.3857/roj.2017.00542 independent negative prognostic factor for OS (Table 4).

For all patients, the median OS rate was 69.1\%. There was no significant difference in $0 S$ rate between the group treated with IFRT and the one with TSEBT ( $p=0.6804$ ) (Fig. 2A). OS was significantly different between groups that had or had not clinically positive lymph nodes at the time of consultation of RT (log rank test, $p=0.043$ ) (Fig. 2B) although it was marginally associated with the detrimental effect on survival $(\mathrm{HR}=4.88,95 \% \mathrm{Cl}, 0.89-26.74 ; \mathrm{p}=0.068)$ in Cox 

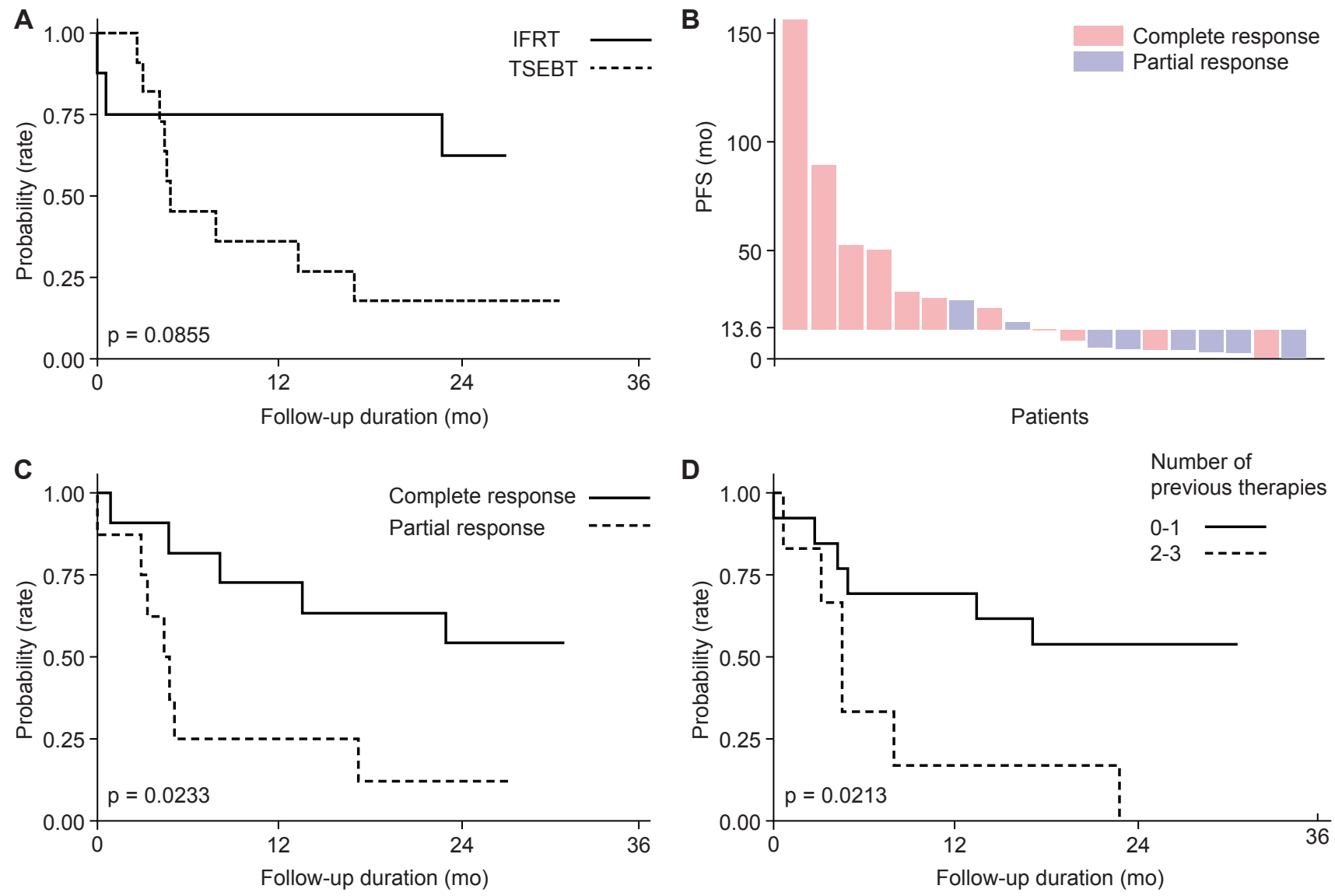

Fig. 1. Kaplan-Meier curves of progression-free survival (PFS) rate for total patients treated with RT (A), for groups stratified by RT responses (C) and by the number of previous therapies (D). Waterfall plot for PFS according to RT responses were depicted in (B). RT, radiation therapy; IFRT, involved field radiation therapy; TSEBT, total skin electron beam radiotherapy. P-values were estimated by logrank test.
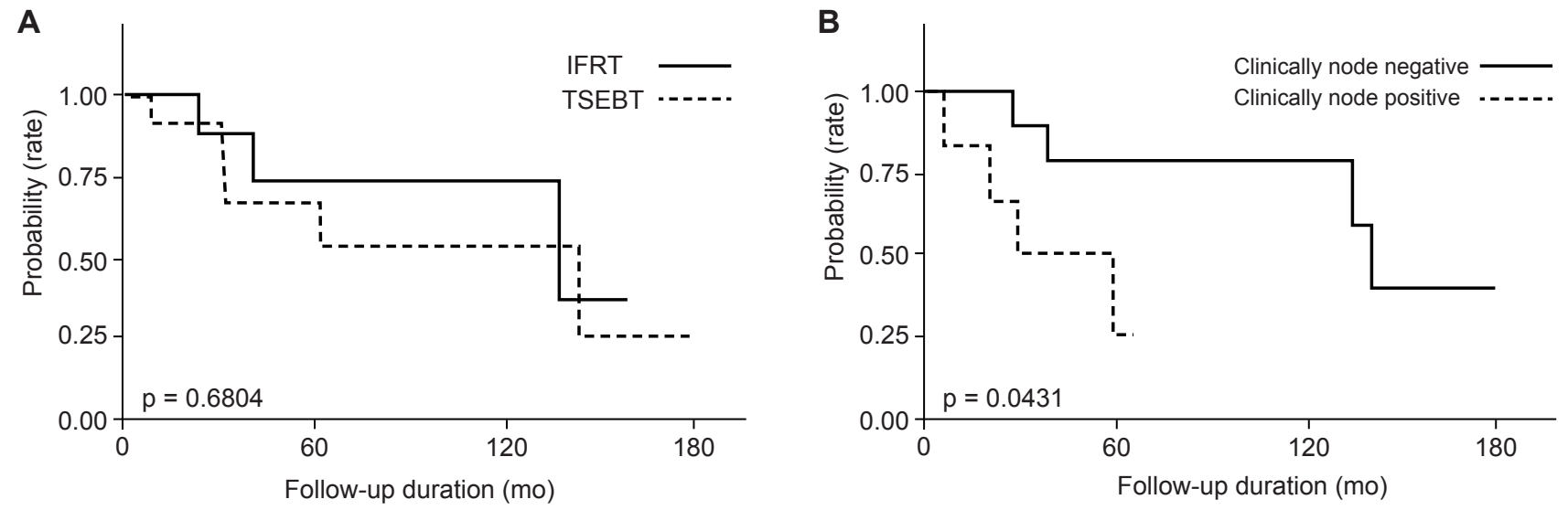

Fig. 2. Kaplan-Meier curves of overall survival rate for total patients underwent radiation therapy (RT) (A) and for groups stratified by the presence of lymph node at the time of consultation of RT. IFRT, involved field radiation therapy; TSEBT, total skin electron beam radiotherapy. P-values were estimated by log-rank test. 
Table 4. Multivariate analysis for progression-free survival, stratified by IFRT/TSEBT

\begin{tabular}{llll}
\hline & \multicolumn{3}{c}{ Progression-free survival } \\
\cline { 2 - 4 } & $H R$ & $95 \% \mathrm{Cl}$ & p-value ${ }^{\mathrm{a})}$ \\
\hline $\begin{array}{l}\text { Number of previous therapies } \\
\quad \text { to } 3 \text { incremental }\end{array}$ & 2.32 & $1.15-4.69$ & 0.019 \\
$\begin{array}{l}\text { Response } \\
\text { CR }\end{array}$ & & & \\
PR & 1.00 & & \\
\hline
\end{tabular}

IFRT, involved field radiation therapy; TSEBT, total skin electron beam radiotherapy; $P R$, partial response; $C R$, complete response; $H R$, hazard ratio; $\mathrm{Cl}$, confidence interval.

${ }^{a)} p$-value was estimated using Cox hazard-proportional regression.

Table 5. Toxicities during or following IFRT/TSEBT $(n=19)$

\begin{tabular}{llll}
\hline & Grade 1 & Grade 2 & Total \\
\hline Nausea & $0(0)$ & $1(0.5)$ & $1(0.5)$ \\
Swelling & $0(0)$ & $3(15.8)$ & $3(15.8)$ \\
Blisters & $1(0.5)$ & $2(10.5)$ & $3(15.8)$ \\
Itching & $1(0.5)$ & $2(10.5)$ & $3(15.8)$ \\
Dry desquamation & $5(26.3)$ & $0(0)$ & $5(26.3)$ \\
Erythema & $0(0)$ & $1(0.5)$ & $1(0.5)$ \\
\hline
\end{tabular}

Values are presented as number of patients (\%).

IFRT, involved field radiation therapy; TSEBT, total skin electron beam radiotherapy.

\section{A}

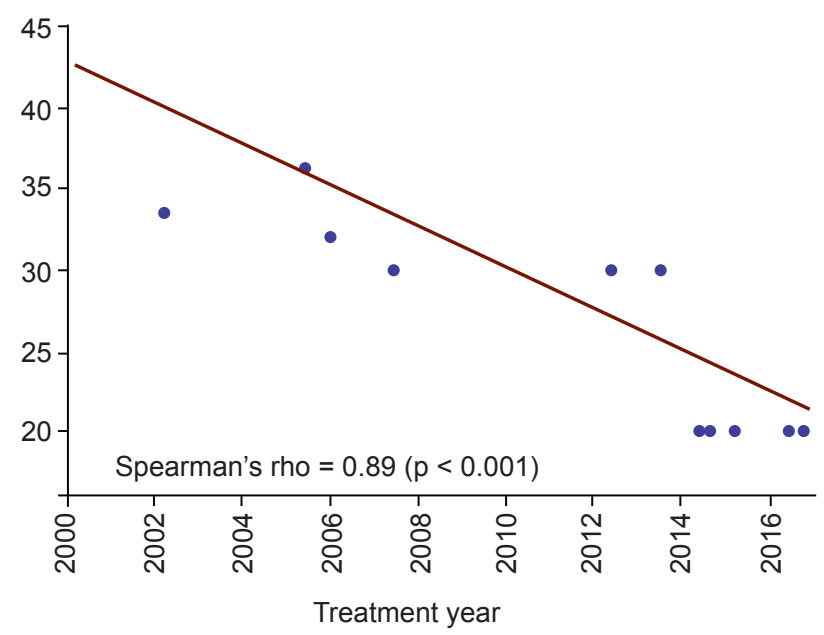

- TSEBT dose (Gy) — Fitted lines proportional-hazard model. Age, gender, the number of previous therapies, radiation doses, RT response, T stage, the administration of adjuvant treatment, time interval between diagnosis and $\mathrm{RT}$, the receipt of treatment had no impact on OS.

We next investigated the trend of prescription dose and the dose-response relationship in our institution. As shown in Fig. 3A, the trend using lower doses for TSEBT over time was observed albeit the most commonly prescribed doses were 20 Gy (Spearman rho $=-0.89, p<0.001$ ). However, we found that there was no significant association between RT responses and RT doses (odds ratio $=0.76, p=0.052$ ). In addition, we examined the effect of various cut-off doses, such as $20 \mathrm{~Gy}$ or $30 \mathrm{~Gy}$, on RT responses, but found no significant relevance (data not shown). Patients showing complete response following TSEBT were more found in the lower doses ( $\leq 30 \mathrm{~Gy}$ ) than the higher doses ( $\geq 32 \mathrm{~Gy}$ ) as shown in Fig. 3B.

\section{Toxicities}

Toxicities were recorded during RT and listed in Table 5. The most common acute adverse effect during RT was grade 1 dry desquamation (5 patients, 26.3\%). Swelling, blisters, itching of skin were acute common side effects of RT. There were no reported grade 3 or more toxicities related to RT.

B

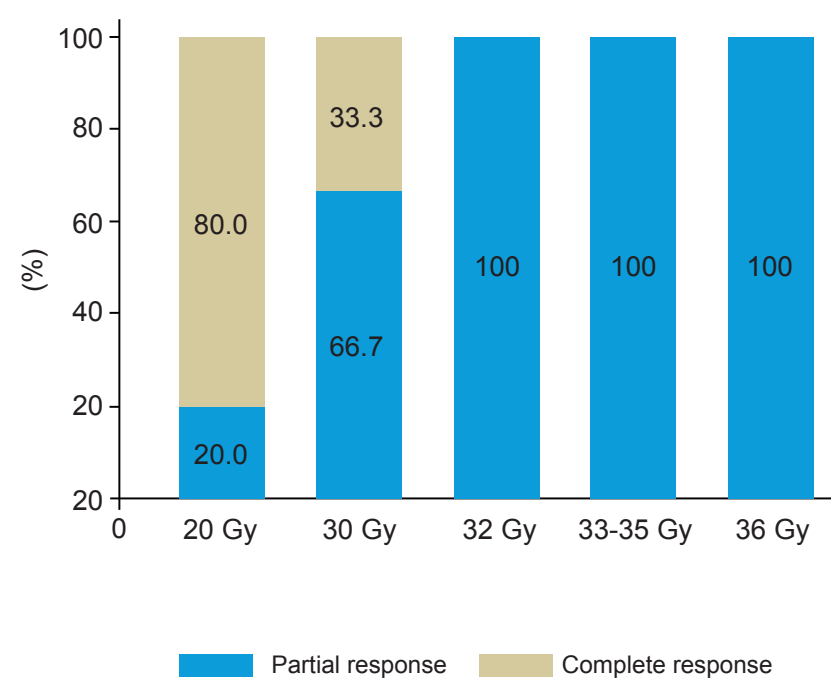

Fig. 3. (A) Graph showing correlation between treatment year and prescribed total skin electron beam radiotherapy (TSEBT) doses. Spearman rank correlation coefficient (Spearman rho) and its p-value are presented. (B) The bar graph showing the proportion of responses to TSEBT doses. 


\section{Discussion and Conclusion}

We showed that the number of previous therapies and RT responses were significant prognostic factor for PFS. In terms of OS, clinically positive lymph node status before RT were found to be important prognostic factor. Kim et al. [12] reported that age, $T$ classification, and the presence of extracutaneous disease were associated with survival. Particularly, advanced T classification was found to be related with a greater risk of progression. Another study [13] addressed that advanced T stage, the presence of tumor clone in peripheral blood, folliculotropic MF were associated with worsened survival and progression. However, our clinical data could not identify that the T stage correlates with PFS or OS. This might be attributed to the possibility that accurate initial T stage information could not be acquired given that patients had already been in an advanced stage after failure of other treatments before RT. Nevertheless, we found the link between $\mathrm{RT}$ responses and the risk of progression, between the number of previous therapies followed by RT and disease progression, and between the presence of positive lymph node and OS.

For TSEBT, generally OR rate is nearly $100 \%$ and $C R$ rate is 40\%-98\% [4,14] depending on extent of MF involvement, which is consistent with our results showing $45 \%$ of $C R$ rate and 100\% of OR rate. For IFRT, previous studies reported that MF patients with stage IA, which accounts for less than $5 \%$ of $M F$, was effectively managed with IFRT showing $95 \%-100 \%$ of $C R$ rate [15]. Aligned with this, patients underwent IFRT in the present study demonstrated $75 \%$ of $\mathrm{CR}$ rate.

Stratified by stage, there exists various therapies ranged from skin-directed therapy such as topical corticosteroids, retinoids, local radiation, or phototherapy to systemic therapies such as TSEBT or chemotherapy. However, welldesigned prospective phase III clinical trials comparing therapies for clinical outcomes are lacking. Nevertheless, most guidelines [16-18] recommend skin-directed therapy for the limited extent of disease and a more aggressive therapy if disease progresses toward the systemic or refractory extent. For this reason, patients often had experienced multiple other treatments followed by RT. Elsayad et al. [19] reported that all patients $(n=45)$ before TSEBT had been administered with a median 5 therapies (range, 1 to 8 therapies). Several studies for IFRT treating cutaneous T-cell lymphoma were reviewed [6] that previous therapies accounted for $46 \%-100 \%$ of patients who would undergo RT. We found that the number of previous therapies was the prognostic factor for disease progression, though Elsayad et al. [19] addressed that it had no significant impact on clinical outcomes. However, it is hard to compare directly between this previous study and our current study because the previous study aimed to compare efficacy of conventional versus low-dose TSEBT regimen.

The increased number of previous therapies before RT indicates long disease course and acquiring treatment resistance from previous failures. Refractory MF and capability of progression to systemic MF may be related with innate or adaptive immune system during disease course. In fact, allogeneic hematopoietic stem cell transplantation [20] or immune check point inhibitor [21] is considered as effective treatment for refractory or relapsed MF. In particular, a patient with refractory cutaneous T-cell Iymphoma had a chimeric cytotoxic T lymphocyte antigen (CTLA4)-CD28 fusion, and the blockade of this chimeric fusion using ipilimumab showed marked response without toxicities $[22,23]$. Nevertheless, to date, TSEBT is effective and palliative treatment with minima systemic toxicity, and excellent local control can be acquired by IFRT [24].

Although we observed acceptable toxicities of less than grade 3 skin adverse effects in TSEBT, another study of a large number of patients [25] reported the experiences of grade 3 skin toxicities. Given the palliative intent of RT and dose-dependent skin toxicities, low-dose TSEBT has been suggested, considering risk versus benefit ratio of RT. A European study [26] reported that the very low-dose as much as $4 \mathrm{~Gy}$ demonstrated only 2.7 months of response duration with a response rate of 88 percent. However, TSEBT with lower doses range of 10 to $<20$ Gy resulted in comparable OR rate and PFS compared with standard dose [27]. A pooled analysis [28] from 3 phase II trials of 33 patient with stage IB to IIIA MF addressed that OR and CR rates of 12-Gy TSEBT were $88 \%$ and $27 \%$, respectively. Most common toxicity was grade 1 dermatitis, and alopecia was temporary which is often irreversible in the 36-Gy dose. Also, low-dose TSEBT gives room for pretreatment and combination with other treatment as well as short duration of treatment. However, as response duration is relatively short, low-dose TSEBT should be used selectively. In this respect, patients with node-positive in the present study may be candidates for low-dose TSEBT because it can provide patients expected for lower OS with comparable symptom relief but much less toxicities compared to conventional TSEBT. In current study, the decreasing trend in doses of TSEBT over the treatment year was observed. Also, patients showing partial response were more found in the group receiving relatively higher doses. However, this should not be interpreted as the causal relationship. Doses were 
prescribed under radiation oncologist's consideration based on the extent of skin lesion or symptom. The wider extent or severe lesion that were likely to show the PR, the higher doses were prescribed. Further studies are needed to elucidate which subgroup would benefit with low-dose TSEBT.

There are several limitations in the present study. First, this is the retrospective study with small number of patient in a single institution. Second, when evaluating staging, TNMB staging was not strictly applied for patients. Given that most MF patients revisit the clinics of the radiation oncology to receive re-RT several times during the rest of their life, a consistent form to evaluate acute or late toxicities is needed. Third, we pooled IFRT and TSEBT patients and analyzed clinical outcomes because the number of patient in each group is small.

In conclusion, TSEBT and IFRT provide excellent response rate with patients with MF. The number of previous therapies representing duration of disease course and $\mathrm{RT}$ responses were associated with PFS. Since patients with positive lymph node status were related with worsened OS, low-dose TSEBT could be considerable option for them.

\section{Conflict of Interest}

No potential conflict of interest relevant to this article was reported.

\section{References}

1. de Coninck EC, Kim YH, Varghese A, Hoppe RT. Clinical characteristics and outcome of patients with extracutaneous mycosis fungoides. J Clin Oncol 2001;19:779-84.

2. Talpur $R$, Singh $L$, Daulat $S$, et al. Long-term outcomes of 1,263 patients with mycosis fungoides and Sezary syndrome from 1982 to 2009. Clin Cancer Res 2012;18:5051-60.

3. Olsen $E_{\text {, Vonderheid }}$, Pimpinelli $N$, et al. Revisions to the staging and classification of mycosis fungoides and Sezary syndrome: a proposal of the International Society for Cutaneous Lymphomas (ISCL) and the cutaneous Iymphoma task force of the European Organization of Research and Treatment of Cancer (EORTC). Blood 2007;110:1713-22.

4. Jones GW, Hoppe RT, Glatstein E. Electron beam treatment for cutaneous T-cell Iymphoma. Hematol Oncol Clin North Am 1995;9:1057-76.

5. Micaily B, Miyamoto C, Kantor G, et al. Radiotherapy for unilesional mycosis fungoides. Int J Radiat Oncol Biol Phys $1998 ; 42: 361-4$.
6. Thomas TO, Agrawal P, Guitart J, et al. Outcome of patients treated with a single-fraction dose of palliative radiation for cutaneous T-cell lymphoma. Int J Radiat Oncol Biol Phys 2013;85:747-53.

7. Wilson LD, Kacinski BM, Jones GW. Local superficial radiotherapy in the management of minimal stage IA cutaneous T-cell lymphoma (Mycosis Fungoides). Int J Radiat Oncol Biol Phys 1998;40:109-15.

8. Kuten A, Rosenblatt E, Dale J, Leviov M, Tatcher M. Total skin electron irradiation: efficacy in early mycosis fungoides. Leuk Lymphoma 1993;10:281-5.

9. Cotter GW, Baglan RJ, Wasserman TH, Mill W. Palliative radiation treatment of cutaneous mycosis fungoides: a dose response. Int J Radiat Oncol Biol Phys 1983;9:1477-80.

10. Funk $A$, Hensley $F$, Krempien $R$, et al. Palliative total skin electron beam therapy (TSEBT) for advanced cutaneous T-cell Iymphoma. Eur J Dermatol 2008;18:308-12.

11. Breneman D, Duvic M, Kuzel T, Yocum R, Truglia J, Stevens VJ. Phase 1 and 2 trial of bexarotene gel for skin-directed treatment of patients with cutaneous T-cell lymphoma. Arch Dermatol 2002;138:325-32.

12. Kim YH, Liu HL, Mraz-Gernhard S, Varghese A, Hoppe RT. Long-term outcome of 525 patients with mycosis fungoides and Sezary syndrome: clinical prognostic factors and risk for disease progression. Arch Dermatol 2003;139:857-66.

13. Agar NS, Wedgeworth $E_{1}$ Crichton $S$, et al. Survival outcomes and prognostic factors in mycosis fungoides/Sézary syndrome: validation of the revised International Society for Cutaneous Lymphomas/European Organisation for Research and Treatment of Cancer staging proposal. J Clin Oncol 2010;28:4730-9.

14. Jones GW, Tadros A, Hodson DI, Rosenthal D, Roberts J, Thorson B. Prognosis with newly diagnosed mycosis fungoides after total skin electron radiation of 30 or 35 Gy. Int J Radiat Oncol Biol Phys 1994;28:839-45.

15. Tandberg DJ, Craciunescu O, Kelsey CR. Radiation therapy for cutaneous T-cell lymphomas. Dermatol Clin 2015;33:703-13.

16. Trautinger $F$, Knobler $R$, Willemze $R$, et al. EORTC consensus recommendations for the treatment of mycosis fungoides/ Sezary syndrome. Eur J Cancer 2006;42:1014-30.

17. Jones GW, Kacinski BM, Wilson LD, et al. Total skin electron radiation in the management of mycosis fungoides: Consensus of the European Organization for Research and Treatment of Cancer (EORTC) Cutaneous Lymphoma Project Group. J Am Acad Dermatol 2002;47:364-70.

18. Whittaker SJ, Marsden JR, Spittle M, Russell Jones R; British Association of Dermatologists; U.K. Cutaneous Lymphoma 
Group. Joint British Association of Dermatologists and U.K. Cutaneous Lymphoma Group guidelines for the management of primary cutaneous T-cell Iymphomas. Br J Dermatol 2003;149:1095-107.

19. Elsayad K, Kriz J, Moustakis C, et al. Total skin electron beam for primary cutaneous T-cell Iymphoma. Int J Radiat Oncol Biol Phys 2015;93:1077-86.

20. Atilla E, Atilla PA, Bozdag SC, et al. Allogeneic hematopoietic stem cell transplantation for refractory mycosis fungoides (MF) and Sezary syndrome (SS). Int J Hematol 2017;106:426-30.

21. Khodadoust $M$, Rook $A H$, Porcu $P$, et al. Pembrolizumab for treatment of relapsed/refractory mycosis fungoides and Sezary syndrome: clinical efficacy in a CITN multicenter phase 2 study. Blood 2016;128:181.

22. Sekulic $A$, Liang WS, Tembe $W$, et al. Personalized treatment of Sezary syndrome by targeting a novel CTLA4:CD28 fusion. Mol Genet Genomic Med 2015;3:130-6.

23. Elenitoba-Johnson KS, Wilcox R. A new molecular paradigm in mycosis fungoides and Sezary syndrome. Semin Diagn Pathol
2017;34:15-21.

24. Hoppe RT. Mycosis fungoides: radiation therapy. Dermatol Ther 2003;16:347-54.

25. Ysebaert L, Truc G, Dalac S, et al. Ultimate results of radiation therapy for T1-T2 mycosis fungoides (including reirradiation). Int J Radiat Oncol Biol Phys 2004;58:1128-34.

26. Kamstrup MR, Specht L, Skovgaard GL, Gniadecki R. A prospective, open-label study of low-dose total skin electron beam therapy in mycosis fungoides. Int J Radiat Oncol Biol Phys 2008;71:1204-7.

27. Harrison C, Young J, Navi D, et al. Revisiting low-dose total skin electron beam therapy in mycosis fungoides. Int J Radiat Oncol Biol Phys 2011;81:e651-7.

28. Hoppe RT, Harrison C, Tavallaee M, et al. Low-dose total skin electron beam therapy as an effective modality to reduce disease burden in patients with mycosis fungoides: results of a pooled analysis from 3 phase-II clinical trials. J Am Acad Dermatol 2015;72:286-92. 